\title{
A Hybrid Uncertainty Propagation Method for System Risk Assessment
}

\author{
Yongsheng Duan ${ }^{1,2, a}$, Tengda Xin ${ }^{2, b}$, Bao Cui ${ }^{3, c}, \mathrm{Na} \mathrm{An}{ }^{2, \text { d }}$, Guoyu Bai ${ }^{4, ~ e ~}$ \\ ${ }^{1}$ Key Laboratory of Space Launching Site Reliability Technology, Haikou, China \\ ${ }^{2}$ Space engineering university, Beijing, China \\ ${ }^{3}$ China Satellite Maritime Tracking and Controlling Department, Jiangyin, China \\ ${ }^{4}$ Jiuquan Satellite Launch Center, Jiuquan, China

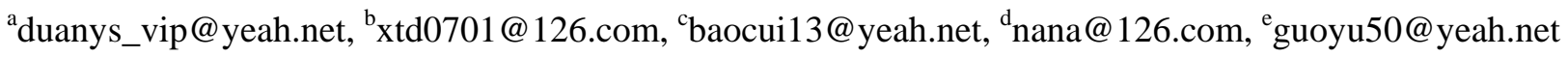

Keywords: Risk assessment, Hybrid uncertainty, Monte Carlo, Epidemic uncertainty.

\begin{abstract}
This paper proposes a hybrid uncertainty propagation approach for system risk assessment. In view of hybrid uncertainty propagation with parameters dependency of variables in risk assessment, a two level hybrid uncertainty propagation framework was proposed, in which inner and outer parameters are characterized with probability and possibility respectively, the numerical values are calculated by Monte Carlo (MC) simulation and fuzzy extension principle. In consideration of the dependency of epidemic uncertainty parameters, an epidemic uncertainty parameter dependency model is designed and a dependency coefficient is proposed. Then the proposed method was compared with the conventional two level MC. Finally, taking the safety of one hydrogen and oxygen co-bottom tank an example, the effectiveness and feasibility of the proposed method was validated.
\end{abstract}

\section{Introduction}

In the field of system engineering, the characterization and propagation of uncertainty in systematic quantitative risk assessment and the influence of uncertainty on risk decision-making are concerned as a hot issue[1, 2]. Especially in aerospace, aviation, nuclear power and other fields, when accurate basic reliability data can't be obtained, risk uncertainty becomes the main basis for risk decision-making.

Risk uncertainty includes two types[3]: One is the stochastic uncertainty (also called objective uncertainty) caused by the randomness and contingency of the internal behavior of the system; the other is the epistemic uncertainty (also called subjective uncertainty) caused by the lack of information and knowledge of the system. In recent years, probability theory as a commonly mean for uncertainty characterization and transmission has been questioned, which can't be used to deal with epistemic uncertainty especially when the data is not accurate, knowledge is not complete. For the characterization of epistemic uncertainty, Moens D[4-6] promote interval method to describe epistemic uncertainty, Rohmer J[7] use probability distribution to depict seismic risk model and data uncertainty, Agarwal H[8] proposed a uncertainty quantification method based on evidence theory for multi attribute optimization design, Shah[9] use evidence theory to model and propagate uncertainty.

When stochastic uncertainty and epistemic uncertainty exist simultaneously in risk model, Guyonnet D[10] proposed a hybrid uncertainty analysis framework, Flage R[11] used a combined method by FT and MC to propagate risk uncertainty of the nuclear reactor thermal loop control system, Baraldi[12] combines ET with MC method to study the risk hybrid uncertainty of nuclear power plant, and the propagation characteristics of uncertainty under probability, possibility and probability-possibility framework are given respectively.

The above method only considers the mixed uncertainties when the risk model input variables, the uncertainties of the distribution parameters of the input variables doesn't been considered in the risk model. Baudrit[13] established a two-layer hybrid uncertainty propagation framework, the epistemic 
uncertainty parameters were completely dependent or completely independent, however the mixed uncertainty propagation was not considered when incomplete dependence between the distribution parameters of variables exists. Therefore, this paper proposes a uncertainty propagation framework considering the interdependence of cognitive uncertainties. For the disadvantage of MC sampling independence or complete dependence of uncertainty, a framework of distributed parameter cognitive uncertainty dependence analysis is proposed, which can deal with the complete dependence, partial dependence and independent propagation of parameters. In order to realize the independent propagation of the distribution parameters, the probability distribution is transformed into the evidence belief space, and the random set theory is used to realize the independent propagation of cognitive uncertainty. Finally, an example is given to illustrate the effectiveness and feasibility of the proposed method.

\section{Possibility Theory}

Possibility theory has the ability to deal with uncertainties when information is inaccurate and knowledge is incomplete. Unlike probability theory (using a single probability measure), possibility theory uses a pair of set functions (possibility measure and necessity measure) to characterize uncertainty[14]. Fuzzy set as classical tool of possibility theory is commonly used to characterize the uncertainty. A brief description of fuzzy sets theory is given here. Details are given in the literature[15].

$\left(a_{1}, a_{2}, \cdots, a_{n}\right)$ are elements of fuzzy set $\tilde{\mathrm{A}}$, membership function $\left(\mu_{i}\right)$ of $a_{i}(i=1,2, \cdots, n)$ characterize the degree of fuzzy set $\tilde{\mathrm{A}}$, the value is in the interval $[0,1][16]$.

Dubois[17] gives the description of membership degree: when $\mu_{\tilde{\mathrm{A}}}(u)=1, u$ totally belong to set A ; when $\mu_{\tilde{\mathrm{A}}}(u)>\mu_{\tilde{\mathrm{A}}}(v)$ meaning the possibility of $u$ is larger than $v$; if there is an element $a_{i}$, and $\mu_{\tilde{\mathrm{A}}}\left(a_{i}\right)=1$, then the fuzzy set $\tilde{\mathrm{A}}$ is normal fuzzy sets.

Under the framework of possibility theory, possibility measure $\Pi$ and necessity $N$ are defined as follows[18]:

Set $U$ is the outputs of $y$, possibility measure $A$ is subset of $U$ and defined as:

$$
\Pi(A)=\sup _{y \in A} \pi(y)
$$

Necessity measure $N(A)$ is defined as:

$$
N(A)=1-\Pi(\bar{A})=\inf _{y \notin A}(1-\pi(y))
$$

$\alpha$-cut set is a interval value $A_{\alpha}=\left\{x \in U, \mu_{\tilde{\mathrm{A}}}(\mathrm{x}) \geq \alpha\right\}$, it shows that the membership degree of each element in the interval is greater than or equal to $\alpha$, shown as in Fig. 1. Lower bound $x_{L}^{\alpha}$ and upper bound $x_{R}^{\alpha}$ of triangular fuzzy distribution $\alpha$-cut set can be calculated as:

$$
\left\{\begin{array}{l}
x_{L}^{\alpha}=a \cdot(b-a)+a \\
x_{R}^{\alpha}=c-a \cdot(c-b)
\end{array}\right.
$$

The principle of fuzzy number $\alpha$-cut set operation shown as Eq.4 and Eq.5

$$
\begin{aligned}
& Z=X \cdot Y= {\left[\min \left(x_{L}^{\alpha} \cdot y_{L}^{\alpha}, x_{L}^{\alpha} \cdot y_{R}^{\alpha}, x_{R}^{\alpha} \cdot y_{L}^{\alpha}, x_{R}^{\alpha} \cdot y_{R}^{\alpha}\right),\right.} \\
&\left.\max \left(x_{L}^{\alpha} \cdot y_{L}^{\alpha}, x_{L}^{\alpha} \cdot y_{R}^{\alpha}, x_{R}^{\alpha} \cdot y_{L}^{\alpha}, x_{R}^{\alpha} \cdot y_{R}^{\alpha}\right)\right] \\
& Z=X+Y=\left[x_{L}^{\alpha}+y_{L}^{\alpha}, x_{R}^{\alpha}+y_{R}^{\alpha}\right]
\end{aligned}
$$




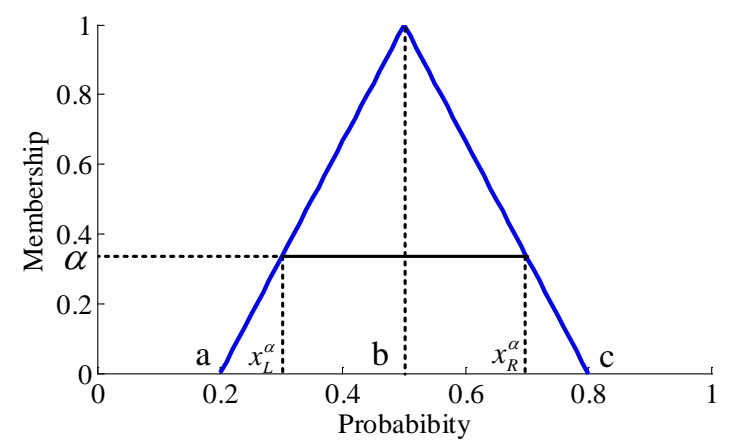

Fig. 1 Interval corresponding to the $\alpha$-cut for the triangular fuzzy membership

\section{Hybrid Uncertainty Propagation Framework Considering Parameter Dependency}

\subsection{Epistemic uncertainty parameters dependency model}

Normally, $\alpha$-cut is used to calculate the possibility distribution, the parameters of epistemic uncertainty are supposed dependent each other completely[19]. However, in actual system engineering, epistemic uncertainty parameters are partially dependent, independent or positive(negative) dependent[20]. Therefore, the concept of the dependence coefficient of cognitive uncertainty parameters is proposed in this paper. The concrete analysis is as follows, detailed analysis is as follows:

$A_{1}^{\alpha}$ and $A_{2}^{\alpha}$ are $\alpha$-cut sets of possibility number $y_{1}$ and $y_{2}$ respectively, $k \in[-1,1]$ is dependency coefficient of two fuzzy numbers, dependency model of $y_{1}$ and $y_{2}$ is defined as Eq.6. When $0<k \leq 1, y_{1}$ and $y_{2}$ is positive dependence; when $-1 \leq k<0, y_{1}$ and $y_{2}$ negative dependent; when $k=0, y_{1}$ and $y_{2}$ independent; specially, when $k= \pm 1, y_{1}$ and $y_{2}$ positive (negative) dependent.

$$
\frac{d y_{1}^{\alpha}}{d \alpha}=k \frac{d y_{2}^{\alpha}}{d \alpha}
$$

\subsection{Hybrid uncertainty propagation with parameters dependency}

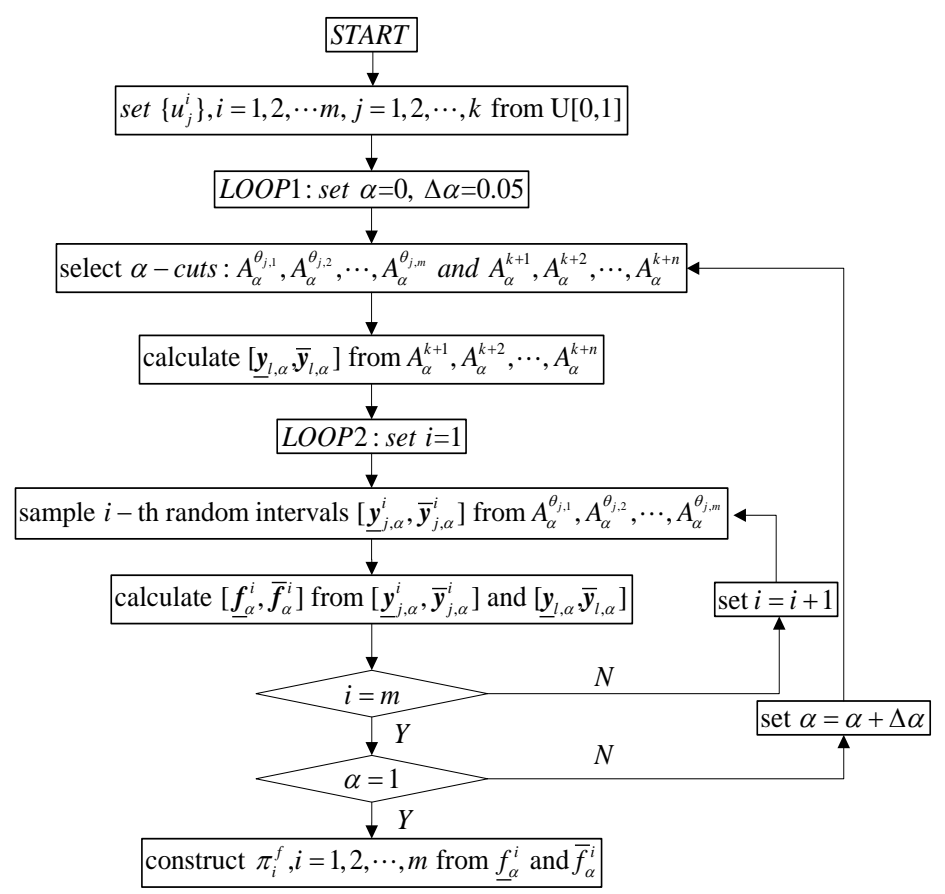

Fig. 2 Hybrid uncertainty propagation flow chart 
$R=f\left(Y_{1}, Y_{1}, \cdots, Y_{n}\right)$ is risk model, $Y_{i}(i=1, \cdots, n)$ is input variable. The first $k$ variables $\left\{Y_{1}, Y_{2}, \cdots, Y_{k}\right\}$ are probability distribution function $p_{Y_{1}}\left(y_{1} \mid \theta_{1}\right), p_{Y_{2}}\left(y_{2} \mid \theta_{2}\right), \cdots p_{Y_{k}}\left(y_{k} \mid \theta_{k}\right)$, and the distribution parameter vector is $\boldsymbol{\theta}_{j}=\left\{\theta_{j, 1}, \theta_{j, 2}, \cdots, \theta_{j, m}\right\}, j=1,2, \cdots, k$, which are probability distributions, denoted as $\boldsymbol{\pi}^{\boldsymbol{\theta}_{j}}\left(\boldsymbol{\theta}_{j}\right)=\left\{\pi^{\theta_{j, 1}}\left(\theta_{j, 1}\right), \pi^{\theta_{j, 2}}\left(\theta_{j, 2}\right), \cdots, \pi^{\theta_{j, m_{j}}}\left(\theta_{j, m_{j}}\right)\right\}$. Variables $\left\{Y_{k+1}, Y_{k+2}, \cdots, Y_{l}, \cdots, Y_{n}\right\} \quad$ are possibility distribution, denoted as $\pi^{Y_{k+1}}\left(y_{k+1}\right), \pi^{Y_{k+2}}\left(y_{k+2}\right), \cdots, \pi^{Y_{l}}\left(y_{l}\right), \cdots, \pi^{Y_{l}}\left(y_{l}\right)$.

Under the two level uncertainty propagation framework, risk uncertainty propagated through MC numerical simulation and fuzzy extension principle. MC realizes probability distribution sampling, and fuzzy sets are used to deal with epistemic uncertainty. Detailed algorithm flow is shown in Fig2.

Flow chart follows as:

(1) Generate random sampling matrix $\left\{u_{j}^{i}\right\}, u_{j}^{i} \in U[0,1], i=1, \cdots, n, j=1,2, \cdots, k, \quad U$ obey uniform distribution;

(2) $\alpha=0$, step of $\Delta \alpha$ is 0.05 , start the outer $L O O P 1$;

(3) Calculate the $\alpha$-cut sets $A_{\alpha}^{\theta_{j, 1}}, A_{\alpha}^{\theta_{j, 2}}, \cdots, A_{\alpha}^{\theta_{j, m}}$ and $A_{\alpha}^{k+1}, A_{\alpha}^{k+2}, \cdots, A_{\alpha}^{k+n}$ of the probability distribution parameter vector $\theta_{j, 1}, \theta_{j, 2}, \cdots, \theta_{j, m}, j=1,2, \cdots, k$ and the possibility distribution parameter vector $Y_{k+1}, \ldots, Y_{l}, \ldots, Y_{n}$, then calculate $\left[\underline{y}_{l, \alpha}, \bar{y}_{l, \alpha}\right], l=k+1, k+2, \cdots, n$ by $A_{\alpha}^{k+1}, A_{\alpha}^{k+2}, \cdots, A_{\alpha}^{k+n}$;

(4) $i=1$, start inner LOOP2;

(5) Calculate the random intervals $\left[\underline{\boldsymbol{y}}_{j, \alpha}^{i}, \overline{\boldsymbol{y}}_{j, \alpha}^{i}\right]$ of probability distribution vectors $\boldsymbol{Y}_{j}, j=1,2, \cdots, k$ by (1) and (3);

(6) Calculate $\left[\underline{\boldsymbol{f}}_{\alpha}^{i}, \overline{\boldsymbol{f}}_{\alpha}^{i}\right]$ by (3) and (5), $\underline{f}_{\alpha}^{i}$ and $\bar{f}_{\alpha}^{i}$ are lower and outer bound of $i$ th random sample $\quad \alpha$-cut set; there is $\underline{f}_{\alpha}^{i}=\inf _{j, Y_{j} \in\left[\underline{I}_{j, \alpha}^{i}, \bar{y}_{j, \alpha}^{i}\right] ; i, Y_{l} \in\left[\underline{y}_{l, \alpha}, \bar{y}_{l, \alpha}\right]} f\left(Y_{1}, Y_{2}, \ldots, Y_{n}\right) \quad$ and $\bar{f}_{\alpha}^{i}=\sup _{j, Y_{j} \in\left[\underline{y}_{j, \alpha}^{i}, \bar{y}_{j, \alpha}^{i}\right] ;, l, Y_{l} \in\left[\underline{y}_{l}, \alpha, \bar{y}_{l, \alpha}\right]} f\left(Y_{1}, Y_{2}, \ldots, Y_{n}\right)$;

(7) If $i \neq m, i=i+1$ and return (5); otherwise, return (8).

(8) If $\alpha \neq 1, \alpha=1$ and return (3); otherwise, construct $m$ possibility distribution functions $\pi_{i}^{f}, i=1,2, \cdots, m$, and end.

After the above processes, the outputs $\pi_{i}^{f}, i=1,2, \cdots, m$ of risk model $Z=f\left(Y_{1}, Y_{2}, \ldots, Y_{n}\right)$ can be obtained, $m$ is the sample times of random variables.

Set $A$ belongs to output $U_{Z}$ of risk model $\mathrm{Z}$, then the possibility measure and necessity measure of set $A$ are

$$
\begin{aligned}
& \prod_{i}^{f}(A)=\sup _{z \in A}\left\{\pi_{i}^{f}(z)\right\} \\
& N_{i}^{f}(A)=\inf _{z \notin A}\left\{1-\pi_{i}^{f}(z)\right\}=1-\prod_{i}^{f}(\bar{A}) \quad \forall A \subseteq U_{Z}
\end{aligned}
$$

In addition, the belief and plausibility measures can be obtained as by $m$ possibility and necessity measures[13].

$$
\begin{aligned}
& \operatorname{Bel}(A)=\sum_{i=1}^{m} p_{i} N_{i}^{f}(A) \\
& \operatorname{Pl}(A)=\sum_{i=1}^{m} p_{i} \Pi_{i}^{f}(A)
\end{aligned}
$$

Here, $p_{i}$ is sample probability of $i$ th stochastic variable $\left(Y_{1}, Y_{2}, \ldots, Y_{j}, \ldots, Y_{k}\right)$, if $m$ is uniform 
sampling, $p_{i}=1 / \mathrm{m}$.

\section{The Uncertainty Analysis of Cryogenic Hydrogen and Oxygen Co-bottom Tank}

\subsection{Cryogenic hydrogen and oxygen co-bottom tank model}

The fuel tank structure of a type of hydrogen-oxygen engine is designed by adiabatic composite material. The hydrogen and oxygen tank is designed by co-bottom structure, and the co-bottom is vacuum sealed space, and the tank is divided into two independent parts: liquid hydrogen tank and liquid oxygen tank. Because the mixture ratio of hydrogen and oxygen reaches a certain condition, it is easy to explode, so the co-bottom is also the safety barrier of the whole tank. To ensure the safety of the tank, a common bottom safety monitoring system is used to detect the pressure, gas concentration and vacuum of the co-bottom[21].

In order to ensure the heat insulation at the bottom, the main technical indicators are: $12 \mathrm{~h}$ leakage rate should be less than $3 \times 10^{-3} \mathrm{~Pa} \cdot \mathrm{m}^{3} / \mathrm{s}$. In this paper, 12 hours static leakage rate is used to characterize the safety of hydrogen and oxygen tank.

$$
Q=\left(P_{1}-P_{2}\right) \cdot V / \Delta T, \Delta T=T_{2}-T_{1}
$$

In Eq.11, $Q$ is co-bottom leakage rate $\left(\mathrm{Pa} \cdot \mathrm{m}^{3} / \mathrm{s}\right), P_{1}$ is co-bottom pressure detection value (Pa) at $T_{1}, P_{2}$ is pressure detection value ( $\left.\mathrm{Pa}\right)$ at $T_{2}, V$ is co-bottom volume $\left(\mathrm{m}^{3}\right), \Delta T$ is time difference (s) between $P_{1}$ and $P_{2}$, inputs $P_{1}, P_{2}, V, T_{1}, T_{2}$ are all uncertainty variables.

\subsection{Uncertainty parameters of co-bottom leakage gas rate}

Due to the influence of systematic errors such as measurement and design, variables $P_{1} 、 P_{2}$ obey normal distribution $P_{1} \sim N\left(\mu_{p 1}, \sigma_{p 1}\right)$ and $P_{2} \sim N\left(\mu_{p 2}, \sigma_{p 2}\right)$, but the distribution parameters can't be obtained accurately. The descriptions of epistemic uncertainty parameters are given by experts in field, variance $\mu_{p 1}$ and $\sigma_{p 1}$ obey fuzzy triangular distribution, standard deviation $\sigma_{p 1}$ and $\sigma_{p 2}$ are constant values. Co-bottom volume $V$ obey lognormal distribution $V \sim \operatorname{LN}\left(\mu_{V}, \sigma_{V}\right) \cdot \mu_{V}$ and $\sigma_{V}$ are epistemic variables, which fuzzy number, $T_{1}$ and $T_{2}$ obey normal distribution $T_{1} \sim N\left(\mu_{T 1}, \sigma_{T 1}\right)$ and $T_{2} \sim N\left(\mu_{T 2}, \sigma_{T 2}\right) \cdot \mu_{T 1}, \sigma_{T 1}, \mu_{T 2}$ and $\sigma_{T 2}$ obey fuzzy triangular distribution. Detailed parameters shown in Table 1.

Table 1 Parameter distributions of epistemic uncertainty

\begin{tabular}{cccc}
\hline Variables & Value & Variables & value \\
\hline$\mu_{p 1}$ & $(6500,7000$, & $\sigma_{V}$ & $(0.0045,0.005$, \\
& $7500)$ & & $0.0055)$ \\
$\sigma_{p 1}$ & 100 & $\mu_{T_{1}}$ & $(0,10,5)$ \\
$\mu_{p 2}$ & $(3300,3500,3400)$ & $\sigma_{T_{1}}$ & $(0.05,0,0.1)$ \\
$\sigma_{p 2}$ & 100 & $\mu_{T_{2}}$ & $(43100,43200,43300)$ \\
$\mu_{V}$ & $(0.49,0.5,0.51)$ & $\sigma_{T_{2}}$ & $(0,100,200)$
\end{tabular}

Due to the influence of subjective preferences, the epistemic uncertainty parameters are interdependent. $\mu_{\mathrm{p} 1}, \mu_{\mathrm{p} 2}, \sigma_{\mathrm{p} 1}, \sigma_{\mathrm{p} 2}, \mu_{\mathrm{T} 1}$ and $\mu_{\mathrm{T} 2}$ are dependent. The other parameters are independent of each other, dependent coefficient are given in Table 2. 
Table 2 Relationships of parameter distributions of epistemic uncertainty

\begin{tabular}{ccc}
\hline Variable1 & Variable2 & Dependent coefficient $k$ \\
\hline$\mu_{p 1}$ & $\mu_{p 2}$ & -0.5 \\
$\sigma_{p 1}$ & $\sigma_{p 2}$ & 0.3 \\
$\mu_{T 1}$ & $\mu_{T 2}$ & -1 \\
$\sigma_{T 1}$ & $\sigma_{T 2}$ & 0.7 \\
\hline
\end{tabular}

\subsection{Results analysis}

1) To verify the validity of the proposed method, a comparison between the proposed method and the 2-level MC method proposed by Baudrit C[13] is made when the parameters are independent. The result is shown in Fig.3. By comparison, the proposed mixed uncertainty propagation method is consistent with the 2-level MC method. The cumulative probability distributions are consistent with Bel and $P l$, and $\operatorname{Bel}(Q<0.03)=0.91, C D F_{\text {uper }}(Q<0.03)=0.9$.

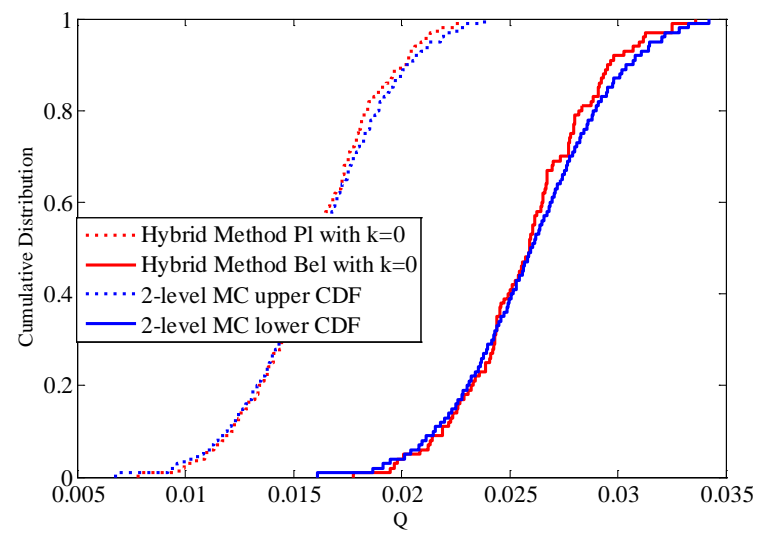

Fig. 3 Curevs of proposed method and Baudrit C's method with epistemic parameters independency

2) In order to analyze the influence of parameter dependence of cognitive uncertainty on the propagation results, taking the $\mu_{\mathrm{p} 1}$ and $\mu_{p 2}$ as the analysis object, assuming that other parameters obey the distribution of in Table 1, when $k=0$ and $k=1$ the results are shown in Fig.4. By contrast, when the epistemic uncertainty parameters are completely dependent,

By contrast, when the cognitive uncertainty parameters are completely dependent, $\mathrm{Bel}_{\mathrm{k}=1}$ and $P l_{\mathrm{k}=1}$ are enveloped by $B e l_{\mathrm{k}=0}$ and $P l_{\mathrm{k}=0}$. Moreover, when the uncertainty parameters are independent, the results of risk uncertainty is more conservative.

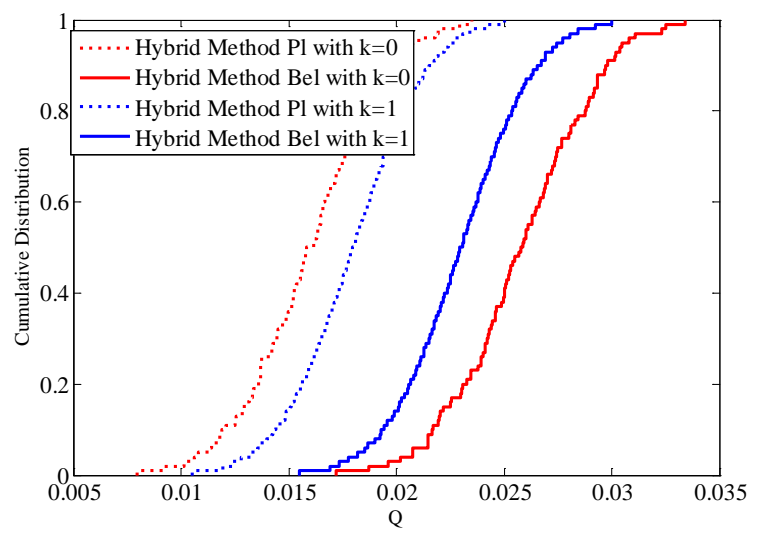

Fig. 4 Curves of hybrid uncertainty propagation with epistemic parameters independent and dependent 


\section{Conclusions}

In this paper a hybrid uncertainty propagation framework considering the parameter dependence of cognitive uncertainty is proposed. Compared with the bi-cyclic MC framework, the effectiveness of the proposed method is verified. At the same time, A general dependency model for epistemic uncertainty parameters is proposed. Especially for parameter independence, a hybrid uncertainty propagation algorithm based on D-S evidence theory is proposed to overcome the problem of high computational cost for dual-cycle MC. When the epistemic uncertainty parameters are characterized by possibility theory, and the parameters are independent, the risk uncertainty is the greatest, which is suitable for the case that the exact probability distribution parameters can not be obtained, and the risk calculation results are relatively conservative.

\section{Acknowledgement}

The authors gratefully acknowledge the financial support on this work from the Key Laboratory of Space Launching Site Reliability Technology of China.

\section{References}

[1] Lindley D V. Understanding Uncertainty[M]. Wiley-Interscience, 2012: 211-224.

[2] Helton J C, Johnson J D, Oberkampf W L, et al. Representation of analysis results involving aleatory and epistemic uncertainty[J]. International Journal of General Systems. 2010, 39(6): 605-646.

[3] Helton J C. Uncertainty and sensitivity analysis in the presence of stochastic and subjective uncertainty[J]. Journal of Statistical Computation \& Simulation. 2007, 57(1): 3-76.

[4] Guo S, Zhen L U, Feng Y. A non-probabilistic model of structural reliability based on interval analysis[J]. Chinese Journal of Computational Mechanics. 2001.

[5] Moens D, Hanss M. Non-probabilistic finite element analysis for parametric uncertainty treatment in applied mechanics: Recent advances[J]. Finite Elements in Analysis \& Design. 2011, 47(1): 4-16.

[6] Qiu Z, Ma Y, Wang X. Comparison between non-probabilistic interval analysis method and probabilistic approach in static response problem of structures with uncertain-but-bounded parameters[J]. Communications in Numerical Methods in Engineering. 2004, 20(4): 279-290.

[7] Rohmer J, Baudrit C. The use of the possibility theory to investigate the epistemic uncertainties within scenario-based earthquake risk assessments[J]. Natural Hazards. 2011, 56(3): 613-632.

[8] Agarwal H, Renaud J E, Preston E L, et al. Uncertainty quantification using evidence theory in multidisciplinary design optimization[J]. Reliability Engineering \& System Safety. 2004, 85(1): 281-294.

[9] Shah H R, Hosder S, Winter T. A mixed uncertainty quantification approach with evidence theory and stochastic expansions[C]. 2014.

[10] Guyonnet D, Bourgine B, Dubois D, et al. Hybrid Approach for Addressing Uncertainty in Risk Assessments[J]. Journal of Environmental Engineering. 2003, 129(1): 68-78.

[11] Flage R, Baraldi P, Ameruso F, et al. Handling epistemic uncertainties in fault tree analysis by probabilistic and possibilistic approaches[C]. 2009.

[12] Baraldi P, Zio E. A combined Monte Carlo and possibilistic approach to uncertainty propagation in event tree analysis[J]. Risk Analysis. 2008, 28(5): 1309-1326.

[13] Baudrit C, Dubois D, Guyonnet D. Joint Propagation and Exploitation of Probabilistic and Possibilistic Information in Risk Assessment[J]. IEEE Transactions on Fuzzy Systems. 2006, 14(5): 593-608. 
[14] Dubois D, Nguyen $\mathrm{H}$ T, Prade H. Possibility theory, probability and fuzzy sets misunderstandings, bridges and gaps[M]. Fundamentals of fuzzy sets, Springer, 2000, 343-438.

[15] Ross T J. Fuzzy logic with engineering applications[M]. John Wiley \& Sons, 2009.

[16] Dubois B P H. Fuzzy Sets and Systems: Theory and Applications[J]. Journal of the Operational Research Society. 1980, 144: 9-146.

[17] Dubois B P H. Fuzzy Sets and Systems: Theory and Applications[J]. Journal of the Operational Research Society. 1997, 144.

[18] Dubois D. Possibility theory and statistical reasoning[J]. Computational statistics \& data analysis. 2006, 51(1): 47-69.

[19] Baudrit C, Dubois D, Guyonnet D. Joint Propagation and Exploitation of Probabilistic and Possibilistic Information in Risk Assessment[J]. IEEE Transactions on Fuzzy Systems. 2006, 14(5): 593-608.

[20] Pedroni N, Zio E. EMPIRICAL COMPARISON OF METHODS FOR THE HIERARCHICAL PROPAGATION OF HYBRID UNCERTAINTY IN RISK ASSESSMENT, IN PRESENCE OF DEPENDENCES[J]. International Journal of Uncertainty Fuzziness and Knowledge-Based Systems. 2012, 20(4): 509-557.

[21] R.Z. Wang, T.H. Sun. Analysis and Measure of Vacuum Character for the Co-bulkhead of the Cryogenic Tanks[J]. Missiles and Space Vehicles. 2002(2): 47-51. (In Chinese) 